\title{
Análisis del concepto de abstracción y su uso en referencia a las relaciones conductuales*
}

\section{An analysis of the concept of abstraction and its use about behavioral relations}

\author{
Carlos-Wilcen Villamil B. \\ ORCID: 0000-0002-3897-085X \\ Centro de Estudios e Investigaciones en \\ Comportamiento, \\ Universidad de Guadalajara, México \\ Luis A. Quiroga-Baquero* \\ ORCID: 0000-0002-9646-9860 \\ Universidad Santo Tomás, Colombia \\ Recibido: 6 de diciembre de 2018 \\ Revisado: 26 de enero de 2019 \\ Aceptado: 4 de abril de 2019
}

\section{Resumen}

El concepto de abstracción ha sido difundido en la literatura conceptual y experimental en el análisis de la conducta desde inicios del siglo XX. Su uso en las aproximaciones derivadas de la teoría del condicionamiento, predominantemente ha sido permeado por categorías de orden operacional, lo cual ha permitido que se considere de forma equivalente o análoga a los conceptos de categorización y formación de conceptos, y que se formulen taxonomías de niveles de abstracción con base en criterios morfológicos. En el presente escrito, se describen las características de dichos usos en la tradición del análisis de la conducta, se identifican algunas insuficiencias conceptuales en torno a su definición con base en categorías operacionales y morfológicas, y se propone como alternativa una definición del comportamiento abstracto y una taxonomía de niveles de complejidad de este comportamiento desde una perspectiva interconductual.

Palabras clave: análisis de la conducta; abstracción; comportamiento abstracto; psicología interconductual.

Artículo de investigación. Cómo citar: Villamil, B. C. W., \& Quiroga-Baquero, L. A. (2019). Análisis del concepto de abstracción y su uso en referencia a las relaciones conductuales. Diversitas: Perspectivas en Psicología, 15(2), 335-351. DOI: 10.15332/22563067.5404

Correspondencia: Carlos-Wilcen Villamil B., correo electrónico: wilcenv@gmail.com; Luis A. Quiroga-Baquero, correo electrónico: luisquiroga@ usantotomas.edu.co. Dirección postal: Cra. 9 n. ${ }^{\circ}$ 61-11, Facultad de Psicología, Universidad Santo Tomás de Aquino, Colombia, Código postal: 110311 


\section{Abstract}

The use of the concept of abstraction has been disseminated within the conditioning theory, predominantly permeated by operational categories, which has allowed it to be considered equivalent to the concepts of categorization and concept formation. Consequently, the taxonomies of levels of abstraction are formulated based on morphological criteria. In the present paper, the characteristics of these uses are described, some shortcomings are identified around their definition based on operational and morphological categories, and an alternative definition of abstract behavior and a taxonomy of complexity levels from a perspective interbehavioral is proposed.

Keywords: behavior analysis; abstraction; abstract behavior; interbehavioral psychology.

Desde las aproximaciones teóricas y empíricas fundamentadas en la teoría del condicionamiento, se ha usado el término abstracción para dar cuenta de un logro conductual caracterizado por un tipo particular de comportamiento bajo el control de estímulos, resultado del establecimiento de contingencias pavlovianas u operantes (Mackintosh, 2000). Predominantemente, la abstracción como término científico en el interior del análisis de la conducta se ha concebido de forma operacional, es decir, que se ha definido en términos correspondientes al vocabulario observacional, como un conjunto de operaciones manipulables y medibles entre sus componentes (las relaciones de contingencia pavlovianas u operantes, los eventos de estímulo y de respuesta, y sus funciones correspondientes) (Ribes, 2003; Shapere, 1989).

En este sentido, las propuestas de niveles de abstracción (e.g., Herrnstein, 1990; Zentall, Galizio \& Critchfield, 2002) han tomado como criterio diferenciador, a las morfologías de los estímulos, de las respuestas o de las relaciones de condicionalidad, en términos topográficos, morfológicos y por ende, operacionales. Con base en esto, los términos abstracción, conceptualización, categorización y formación de conceptos, se tornan equivalentes semántica y operacionalmente, como un tipo particular de comportamiento.

Sin embargo, la descripción del comportamiento abstracto no puede descansar exclusivamente en criterios morfológicos (Ribes, 1994a; Robinson \& de Oliveira, 2018), sino que resulta necesario un criterio funcional que permita definir este tipo de comportamiento y los niveles de complejidad en los cuales puede organizarse. Las propuestas teóricas de Ribes y López (1985) y Ribes (2010) pueden constituir una alternativa explicativa en este sentido, ya que abordan la descripción del comportamiento psicológico en términos de campos de relaciones de contingencias (de ocurrencia y de función), que contemplan los criterios morfológicos como condiciones necesarias para el establecimiento de relaciones de condicionalidad, pero es la integración funcional de los segmentos de estímulo y de respuesta los que definen la complejidad del comportamiento.

Así, el presente escrito tiene como objetivo abordar lo que desde la teoría del condicionamiento se ha concebido como comportamiento abstracto y los criterios sobre los cuales se han propuesto diferentes niveles de complejidad de este comportamiento, identificando la insuficiencia de utilizar las propiedades morfológicas como criterio taxonómico. Como alternativa, se propone definir el comportamiento abstracto desde el sistema teórico propuesto por Ribes y López (1985), con el fin de identificar distintos niveles de organización de dicho comportamiento bajo criterios funcionales.

\section{Comportamiento abstracto en el análisis de la conducta}

El término abstracción tiene su origen latín en el prefijo abs (separar), tractus/trahere (arrastrado/ tirado) y el sufijo -ción (acción o efecto) (Patridge, 2006). El diccionario de psicología de la American Psychological Association (VandenBos, 2007), define 
el término abstracción como la formación de ideas generales o conceptos, mediante la extracción de similitudes de casos particulares. El uso de este término parece ser equivalente con las nociones de conceptualización y categorización. La primera, definida como el proceso de formación de conceptos, particularmente aquellos de naturaleza abstracta a partir de la experiencia o el material aprendido; y la segunda, como el proceso por el cual objetos, eventos, personas o experiencias son agrupados en clases sobre la base de (a) características compartidas por los miembros de la misma clase, y (b) características que diferencian los miembros de una clase de aquellos de otra. Dicha equivalencia se puede identificar en tanto que estos tres términos, al compartir el sufijo -ción, suponen una acción, como proceso psicológico relacionado con la extracción de propiedades compartidas por los miembros de una clase o agrupación de eventos concretos, y un efecto comprendido como el resultado de dicho proceso; a saber, un concepto referido a una clase de objetos o sus propiedades (VandenBos, 2007).

Al interior del análisis experimental del comportamiento (AEC) es posible identificar esta misma equivalencia, en tanto que los términos de abstracción, conceptualización y categorización, son interpretados operacionalmente como comportamientos caracterizados por una generalización intra clases y una discriminación entre clases (Keller \& Schoenfeld, 1950), a través del establecimiento de contingencias respondientes u operantes, comprendidas como relaciones de condicionalidad entre eventos de estímulo y de respuesta (Catania, 2007). En otras palabras, el término abstracción se refiere al logro consistente en responder de forma semejante a una clase de objetos que comparten una o varias propiedades (Keller \& Schoenfeld, 1950; Zentall, et al., 2002; Zentall, Wasserman, Lazareva, Thompson \& Ratterman, 2008).

En referencia explícita al concepto de abstracción, Hull (1920) abordó los aspectos cuantitativos de la evolución de conceptos generales, a partir de la abstracción de elementos comunes en un conjunto o clase de instancias, identificando tres formas de abstracción generalizante. En la primera, una repuesta se transfiere de un objeto de estímulo a otro en la medida en que comparten un elemento común (e.g., luminosidad), el cual en principio fue extraído del complejo bruto de la situación primaria en que tuvo lugar. La segunda, y más cercana a la concepción tradicional de conducta conceptual (Keller \& Schoenfeld, 1950), implica que una respuesta (en algunos casos lingüística) ante un elemento, surge de la comparación de similitudes y diferencias entre diversas situaciones que contienen en común dicho elemento. La última, y base de su abordaje acerca del proceso natural o estándar para la abstracción y evolución de los conceptos, se presenta cuando una respuesta común surge de diversas situaciones que comparten elementos, pero en la cual cada situación está vinculada a un objeto de estímulo que no comparte elementos con la diversidad de situaciones (e. g., una palabra); cada nueva relación situación-objeto de estímulo, es un problema más o menos "agudo" al igual que la reacción adecuada.

Esta última forma de abstracción, metodológicamente se instauró en experimentos con clases conformadas por letras del alfabeto chino, definidas por un trazo común y una sílaba sin sentido, en donde se afirmaba que un individuo había aprendido satisfactoriamente un concepto si era capaz de categorizar nuevos elementos de acuerdo con el trazo y la sílaba. Propuesto así, este último proceso de abstracción generalizante comprendía cuatro aspectos fundamentales: 1) la tendencia a reaccionar de una forma particular a una clase de estímulos que compartían un elemento en común; 2) esta tendencia es establecida por la exposición a aciertos y errores, y sensible a las oscilaciones en la atención, lo que permite responder a las semejanzas y diferencias entre los estímulos, (e .g., Hull, 1920, p. 50, Experimento $\mathrm{G}$ ); 3) el aumento progresivo en la precisión del responder discriminado en las situaciones de entrenamiento y de prueba (ilustrado en las curvas de mejoramiento); y 4) el proceso de ajuste a las condiciones de la tarea, que aunque requería del uso del lenguaje, se daba inconscientemente de tal forma que no fuera guiado de forma intencional.

Teniendo en cuenta esto, el uso del término abstracción en la formulación de Hull se refiere, operacionalmente, a la precisión de las respuestas de categorización de las instancias, en las cuales, el ahorro representado en las curvas asintóticas 
de desempeño permitía medir el aprendizaje, como logro, del concepto.

Por su parte, Skinner (1957) abordó el término abstracción como un tipo de operante verbal denominada tacto abstracto, que resulta de contingencias de reforzamiento diferencial, cuyo efecto es restringir o agudizar el control de una propiedad de estímulo presente en diversos objetos, ante la variación de otras propiedades. Por ejemplo, se puede afirmar que un niño abstrae la propiedad de rojo al decir la palabra rojo ante diversos objetos que comparten dicha propiedad (como longitud de onda de reflexión de la luz) y en los cuales las propiedades de forma, textura o tamaño varían.

No obstante, los tactos abstractos no surgen solamente como eventos verbales en tanto palabras ante objetos, propiedades o acontecimientos, sino que también surgen como eventos verbales en la forma de reglas que se abstraen al estar en contacto con las contingencias que afectan la conducta (Skinner, 1966). Así, por ejemplo, las contingencias naturales que permiten avivar el fuego en la herrería mediante un fuelle: "muy arriba, muy abajo, rápido arriba, lento abajo, y esa es la manera de soplar" (p. 233). En este caso el término abstracto correspondería a una descripción verbal de la conducta bajo el control discriminativo preciso de las propiedades de estímulo que se definen pertinentes.

De esta manera, se equipara a la abstracción con un control de estímulo agudo que se ejerce sobre un tipo respuesta, peculiarmente en un ambiente verbal que proporciona contingencias restringidas, y que es resultante del reforzamiento diferencial. Así, la abstracción se predica de un logro conductual que consiste en un responder discriminado ante una propiedad funcional de eventos particulares o contingencias específicas, del conjunto total de propiedades variantes que constituyen en conjunto la situación estimulativa.

Por su parte, Goldiamond (1966) concibió a la abstracción como un estado final en el que el organismo "está comportándose como si le fueran impuestos sobre sí mismo un conjunto de estímulos [discriminativos] instruccionales, que gobiernan las dimensiones sobre las cuales él responderá con respecto de un estímulo [discriminativo] dimensional" (pp. 199-200). La función discriminativa instruccional de los estímulos es la de restringir las alternativas de respuesta a una dimensión sobre la cual son funcionales otros estímulos, es decir, determina el cómo debe responderse, mientras que la propiedad dimensional se refiere a las propiedades físicas de esos otros estímulos a los cuales el responder diferencial debe limitarse 0 , en otras palabras, determina a qué debe responderse (Layng, Sota \& León, 2011).

El estímulo instruccional y el estímulo dimensional son discriminativos ya que sus funciones se deben a las consecuencias, esto implica que surgen de la exposición a contingencias de reforzamiento, excepto cuando la función instruccional es declarada de manera previa a la experiencia, típico de las situaciones con sujetos humanos (Goldiamond, 1966). De hecho, Goldiamond plantea que la conducta de un animal está bajo control de la regla, o que el animal ha abstraído la regla responda al rojo, cuando se ha reforzado ante objetos de color rojo y no se ha reforzado ante objetos con color no rojo, independientemente de cualquier otra característica física; es decir, que se afirma del estado final del comportamiento a partir del reforzamiento diferencial.

Según este planteamiento, el logro como comportamiento abstracto se predica del ajuste del responder discriminado a las relaciones de condicionalidad, ante la variación en las propiedades físicas de los objetos de estímulo, lo cual puede darse en instancias de discriminación simple en las cuales el responder diferencial del organismo se ajusta a un criterio prescrito por las contingencias de reforzamiento (función instruccional) bajo condiciones de estimulación presentes que ejercen control sobre tal conducta (función dimensional); así como en instancias de discriminación condicional o de control instruccional verbal, en las cuales las condiciones experimentales suponen una separación explícita de las funciones instruccionales y dimensionales (Goldiamond, 1966).

Adicionalmente, es posible identificar el uso del término abstracción en el campo de estudio de la equivalencia de estímulos (Sidman, 1994; Zentall \& Smeets, 1996) y de los marcos relacionales (Hayes, Barnes-Holmes \& Roche, 2001). En el primer caso, 
Sidman y Tailby (1982) y Sidman (1994) utilizaron la noción matemática de relaciones de equivalencia y sus propiedades de reflexividad, simetría y transitividad para describir algunos hallazgos experimentales en discriminaciones condicionales con sujetos humanos y no humanos, en los cuales se presentaba una intercambiabilidad funcional entre estímulos que producían el mismo efecto sobre el comportamiento. El establecimiento de las relaciones de equivalencia se infiere entonces a partir del establecimiento de relaciones de condicionalidad directamente entrenadas y de la emergencia de relaciones no entrenadas, permitiendo así la identificación de clases de equivalencia, comprendidas como conjuntos de estímulos con propiedades funcionales similares.

Sidman (2000) planteó que las relaciones de equivalencia son un resultado directo de las contingencias de reforzamiento de cuatro términos, de tal manera que las propiedades conductuales a partir de las cuales se identifica una clase equivalente de estímulos, corresponden con las propiedades abstractas que en la teoría matemática de conjuntos definen una relación de equivalencia, a saber: relaciones de reflexividad, simetría y transitividad.

Propuesto de esta manera, Sidman (1994) al abordar la formación de las relaciones de equivalencia, planteó que las discriminaciones particulares dan lugar a la abstracción de propiedades comunes usualmente expresadas en palabras, y que, a partir de estas palabras se derivan abstracciones de orden superior, como un proceso de pensamiento que en sí mismo llega a estar disponible por medio de relaciones de equivalencia. En este caso, el uso del término abstracción se da como un logro, como el resultado de un proceso lineal de control de estímulos en el cual se separan las propiedades comunes de un conjunto de objetos, las cuales guardan relaciones de equivalencia con una palabra y por ende, harían parte de la misma clase funcional.

Por su parte, en la Teoría del Marco Relacional (Hayes et al., 2001) se ha usado el término abstracción para referirse a diferentes patrones de comportamiento relacional generalizado, o de segundo orden, caracterizados por: (a) su emergencia a partir de la exposición a múltiples contingencias de reforzamiento, en las que cada instancia de responder discriminativo se presenta ante la correspondencia entre propiedades formales o no formales de los eventos en relación, y en donde todas aquellas instancias en conjunto presentan las misma correspondencia; (b) todas las instancias de responder discriminativo, ocurren en presencia de ciertas claves contextuales que adquieren control sobre el responder relacional; (c) una vez que el responder relacional queda bajo el control de claves contextuales, tanto en las relaciones directamente entrenadas, como en las relaciones derivadas (implicación mutua e implicación combinatoria), entonces el responder relacional es arbitrariamente aplicable a nuevas instancias de propiedades en relación.

En este caso, el término abstracción es usado como un término de logro, ya que se refiere a la discriminación de una relación entre propiedades o atributos de dos o más estímulos, prescrita por la funcionalidad de una clave contextual. Cabe resaltar que esta forma particular de comportamiento relacional, al concebirse como una operante generalizada al igual que el seguimiento de instrucciones o la imitación generalizada, no es definida por características topográficas de los eventos que se relacionan y por lo tanto, puede ser arbitrariamente aplicable a diferentes tipos de propiedades topográficas entre los estímulos (Hayes et al., 2001).

Por ejemplo, el responder relacional a la identidad o semejanza entre dos estímulos bajo el control de una clave contextual (e. g., la palabra igual), denominada como marco de coordinación, podría enmarcar o relacionar como iguales a un perro $y$ a una vaca en términos de la cantidad de patas, o un televisor y un tablero en términos de su forma. En estos casos, la funcionalidad de la clave contextual permite discriminar las propiedades de los estímulos que entran en relación, así los estímulos y sus propiedades topográficas cambien de un caso a otro.

A partir de lo expuesto en este apartado en torno al uso más sobresaliente del término abstracción y su correspondencia funcional con los términos de concepto, categorización y comportamiento conceptual al interior del análisis de la conducta, se ha evidenciado un uso común en términos de la definición ofrecida por Keller y Schoenfeld (1950); 
a saber, que se habla de conducta conceptual cuando se generaliza intra clases y se discrimina entre clases.

En este sentido, el concepto de comportamiento abstracto, al referirse a una forma de responder bajo el control de una propiedad funcional aislada o separada de las relaciones respecto del conjunto total de propiedades o relaciones variantes que constituyen una situación de estímulo, como resultado del establecimiento de las relaciones de condicionalidad prescritas, denota su uso como un término de logro. Los términos de logro se refieren a aquellos términos que indican directamente resultados, efectos o productos, y al predicarlos se está "afirmando que se da algún estado de cosas, además del que consiste en la realización, si es que la hay, de la actividad subordinada" (Ryle, 1949, p. 153).

\section{Niveles de abstracción en el análisis de la conducta}

El término abstracción se ha usado para referirse a un logro conductual, consistente en una respuesta discriminada ante propiedades constantes o comunes a una clase de estímulos, y así, ha sido equivalente con el uso que se hace del término de conducta conceptual. En consonancia con esto, se han propuesto diferentes niveles para clasificar el comportamiento abstracto, bajo el supuesto de que hay diferencias en términos de la relación entre propiedades de estímulos definitorios de una clase a la que un organismo puede responder (Herrnstein, 1990; Zentall et al., 2002).

Herrnstein (1990) afirma que es posible proponer una taxonomía de niveles de control de estímulos que pueden ser descritos con base en un criterio ascendente de abstracción y que permitirían abordar lo que desde otras aproximaciones ha sido concebido como procesos de categorización. Para Herrnstein (1990), la abstracción es más o menos compleja dependiendo de los eventos físicos a los que se responde, pues las propiedades de los objetos se pueden caracterizar por su presencia, ausencia, o concatenación, y estas características guardan relación con las respuestas, las consecuencias y los sistemas perceptuales.
En concreto, propone cinco niveles de comportamiento abstracto: 1) discriminación, que es responder a una variación pequeña de los estímulos, por ejemplo, ante una longitud de onda particular; 2) categorización por memoria, como responder a objetos que son miembros de una clase pequeña los cuales pueden ser semejantes (e. g., los comodines de una baraja) o diferentes (e. g., los nombres de las paradas del metro); 3) categoría abierta-cerrada, que implica responder a una clase de estímulos muy grande con subgrupos establecidos por diferencias y semejanzas perceptuales, por ejemplo, responder a la clase nuez y a las subclases bellota y hayuco; 4) concepto, que implica responder a clases de objetos que no poseen ninguna propiedad perceptual en común, como cuando se responde ante la palabra bebé y la palabra cuna como miembros que van juntos; y 5) relaciones abstractas, que es responder a relaciones entre conceptos, como por ejemplo cuando se responde a "bellotas sobre troncos o papas del mismo color", en donde las palabras sobre y mismo identifican la relación entre conceptos, y la relación no se aplica a una instancia particular si no a cualquier instancia.

La segunda gran clasificación del comportamiento abstracto (e. g., Huber \& Aust, 2006; Zentall et al., 2002; Zentall et al., 2008), propone que la categorización puede ser entendida como un patrón de respuestas diferenciales y sistemáticas a clases de estímulos no-idénticos aunque potencialmente discriminables, y dependiendo de las relaciones entre los objetos, sus propiedades y las consecuencias del responder, es posible identificar cuatro niveles diferenciables en términos de la complejidad del responder discriminativo: 1) aprendizaje de conceptos perceptuales, en el cual las clases de estímulos se establecen con base en un criterio de similitudes perceptuales en los objetos de estímulo; 2) conceptos asociativos en los que el organismo ajusta su comportamiento a clases definidas por propiedades arbitrarias de los objetos; 3) conceptos relacionales, en donde las clases se definen ya no por propiedades absolutas, sino por propiedades relacionales (e. g., semejanza/diferencia); y 4) razonamiento analógico, en el cual el responder es condicional a relaciones entre relaciones.

Es posible identificar tres criterios sobre los que se fundamentan estas taxonomías de niveles de 
abstracción: (a) el responder discriminado se establece por la exposición directa a contingencias de reforzamiento, en tanto relaciones de condicionalidad espacio-temporal entre eventos de estímulo y de respuesta; (b) dicha exposición resulta en el establecimiento de propiedades funcionales discriminativas sobre propiedades físicas de los estímulos; y (c) el continuo de complejidad sobre el cual se diferencian los distintos niveles de abstracción, inicia en la similitud de carácter perceptual entre propiedades físicas, hacia la arbitrariedad en las relaciones condicionales entre estímulos bajo el control de claves de tipo contextual (verbales o no).

El problema con estas concepciones de los niveles de comportamiento abstracto es de dos tipos. Primero, sugiere una progresión en la complejidad de la conducta abstracta y, como en el caso de Herrnstein (1990), una inclusión progresiva de los niveles; no obstante, los criterios de diferenciación y clasificación del comportamiento abstracto tienden a ser morfológicos como predicados de las propiedades físicas de los objetos, o cuantitativos respecto de la cantidad de elementos que pueden conformar una clase. Los criterios morfológicos y cuantitativos no permiten establecer una progresión en complejidad pues no existe un criterio para afirmar que la naturaleza de una propiedad u otra, es más o menos abstracta. Tampoco permiten establecer cómo un nivel se incluye en otro, o cómo un nivel menos complejo precede o se incluye en un nivel posterior o más complejo.

Segundo, y correlativo al primer problema, si bien se resalta el hecho de que los niveles de comportamiento abstracto surgen en las contingencias de reforzamiento, no se especifica si el proceso implicado en el desarrollo de un tipo de comportamiento abstracto es diferente del proceso que tiene lugar en otro nivel de abstracción. En la teoría del condicionamiento sólo existen dos procesos, el operante y el respondiente (Skinner, 1937, 1938). El primer proceso implicaría que el comportamiento abstracto surge como un proceso de correlaciones respuesta-reforzador condicionales a alguna propiedad constante de los estímulos. El segundo proceso se presenta como correlaciones estímulo-estímulo, e implicaría para el comportamiento abstracto que sólo se establecen correlaciones del reforzador con una propiedad constante de los estímulos.
El que se plantee que los niveles de abstracción surgen a partir de las contingencias de reforzamiento, parece indicar que los procesos se reducen sólo a una de las dos clases de condicionamiento. Sin embargo, se ha argumentado que estos dos tipos no representan niveles diferentes de complejidad dado que su diferenciación parte de la relación temporal del estímulo reforzador respecto de la respuesta (Schoenfeld, 1972), por lo cual son niveles horizontales y no verticales, y por lo tanto no se pueden predicar niveles de complejidad e inclusión (Ribes, 1994b; Ribes \& López, 1985).

Ribes (2006) afirma que en la tradición conductual "los conceptos constituyen repertorios discriminativos complejos que establecen una correspondencia funcional con propiedades o atributos definitorios de clases de estímulos" (p. 89). Resaltar el contacto o dependencia funcional entre eventos de respuesta y eventos de estímulo que definen una clase, implica resaltar que éstos sostienen relaciones como integración funcional (Ribes, 2004), las cuales no se limitan a las dependencias en tiempo y espacio entre propiedades morfológicas de los estímulos, tal como se ha concebido en la tradición conductual.

Ribes, Torres, Barrera y Ramírez (1995) ofrecen un ejemplo de lo anterior, al caracterizar los cambios en las propiedades conductuales correlativas a las funciones discriminativa o delta de los objetos en los procesos de discriminación condicional, como "una contingencia de abstracción, en la que el individuo tiene que responder a propiedades de los estímulos presentes, en términos de una propiedad constante que es relacional" (p. 116), en donde, la relación constante entre los estímulos de muestra y de comparación es considerada como control abstracto del estímulo (Ribes, 2000).

Si la contingencia, como relación de dependencia funcional entre eventos de respuesta y eventos de estímulo que definen una clase, puede ser considerada como abstracta, se permite reconocer que existen diferentes niveles de abstracción de acuerdo con la organización de los elementos que participan en una situación, y de la independencia del responder discriminativo en relación con las propiedades físicas que definen la clase. Planteado así, el poder establecer niveles de abstracción debe dirigirse a 
identificar tipos de relaciones o contingencias entre los eventos y el comportamiento y las funciones que resultan, más que a las propiedades morfológicas de los estímulos.

\section{Una taxonomía funcional del comportamiento abstracto}

A partir del análisis de campo propuesto por Ribes y López (1985) y Ribes (2010), es posible identificar una alternativa teórica y empírica que permite describir niveles funcionales de comportamiento abstracto, cuyo criterio taxonómico no descansa en las propiedades físicas perceptuales de los objetos que conforman una clase y sus posibles relaciones arbitrarias o no arbitrarias, ni en la morfología de las relaciones de condicionalidad a partir de las cuales se establecen dichas clases, sino como niveles cualitativamente distintos de comportamiento que se diferencian en su complejidad. A partir de la descripción de dos tipos de relaciones de condicionalidad entre los segmentos de estímulo y de respuesta que participan en una interacción: las contingencias de ocurrencia y las contingencias de función (Ribes, 2007).

En concreto, las contingencias de ocurrencia se refieren a las relaciones de condicionalidad entre la presentación de un evento en relación con la presentación de otro, mientras que las contingencias de función describen la condicionalidad de una propiedad funcional de un estímulo o respuesta, con respecto de la propiedad funcional de otro(s) estímulo(s) o respuestas(s) (Ribes, 2010). Con relación a las primeras, es posible identificar tanto el tipo de organización de las dependencias diacrónicas entre eventos de estímulo, y entre éstos y los eventos de respuesta, así como el tipo de mediación, referido a cómo un evento de estímulo o de respuesta se torna crítico para que dichas relaciones de ocurrencia se articulen. Con relación a las segundas, se identifica el grado de independencia de la reactividad del organismo respecto de las propiedades físicas de los estímulos y de los parámetros espacio-temporales de su ocurrencia (desligamiento funcional), lo que permite algún tipo de ajuste del comportamiento al criterio determinado por la circunstancia de interacción.
Desde esta postura, se han propuesto cinco niveles de organización de campos de contingencias de ocurrencia y de función cualitativamente distintos en términos del tipo de desligamiento funcional y del proceso de mediación implicado; a saber, interacciones contextuales, suplementarias, selectoras, sustitutivas referenciales y sustitutivas no referenciales. Estas estructuras $u$ organizaciones conductuales son posibilitadas por un medio de contacto (físico-químico, ecológico o convencional) y con probabilidades que dependen de los factores disposicionales (factores situacionales e historia interconductual) (Ribes \& López, 1985).

Con base en esto, es posible identificar niveles de interacción abstracta más o menos complejos resultantes de diferentes condicionalidades de ocurrencia y de función; en los tres primeros niveles es posible situar conceptual y empíricamente, los fenómenos conductuales descritos en la tradición de la teoría del condicionamiento, bajo el nombre de conceptualización, categorización y formación de conceptos, como equivalentes del término abstracción. Dicho comportamiento abstracto se identifica con el responder de forma independiente respecto de la concreción o la particularidad de objetos o de relaciones entre sus propiedades, cuando la situación implica la reactividad a la constancia de ciertas propiedades compartidas por un conjunto de objetos, ante la variación de propiedades no relevantes en las relaciones de condicionalidad prescritas. A continuación, se presenta una descripción genérica del comportamiento abstracto en estos tres niveles, complementado con la descripción de situaciones empíricas.

\section{Abstracción en función contextual.}

El comportamiento contextual abstracto se refiere al establecimiento de contingencias de función derivadas del contacto con propiedades absolutas e invariantes de los segmentos de estímulo, entre arreglos variantes. Las contingencias de ocurrencia en este caso, se caracterizan por: (a) una inconstancia relativa respecto de sus propiedades físico-químicas entre los estímulos contingentes en tiempo y espacio que no es afectada por algún seg- 
mento de respuesta (Ribes \& López, 1985); (b) una constancia en los parámetros espacio-temporales y probabilísticos de sucesión de dichos estímulos y segmentos de respuesta; y (c) la variación del remanente de propiedades físicas de los estímulos que no son contingentes.

Por su parte, las contingencias de función se caracterizan por un isomorfismo en la funcionalidad de los segmentos de estímulo y respuesta contextualizados, respecto de las propiedades funcionales de los segmentos de estímulo y respuesta contextualizadores, y de la constancia en los parámetros de ocurrencia de aquellos. El ajuste se da en la forma de respuesta diferencial a los cambios espacio-temporales de los objetos de estímulo, los cuales tienen como característica una constancia total respecto de sus propiedades funcionales.

En un fenómeno relativo a la formación de clases de equivalencia, se usó un procedimiento respondiente para el establecimiento de las clases y uno de igualación a la muestra para su evaluación (Gutiérrez \& Benjumea, 2003). En la fase de entrenamiento se presentaban tres conjuntos de tres figuras arbitrarias cada uno: las figuras del primer conjunto mantenían una contingencia positiva con el sonido de un teléfono (señalaban su ocurrencia), las del segundo conjunto mantenía una contingencia nula (no señalaban ni ocurrencia ni ausencia) y las del tercer grupo una contingencia negativa (señalaban su ausencia). En la fase de prueba, se presentaron arreglos de igualación a la muestra en los cuales el estímulo muestra y uno de los comparativos correspondían al mismo conjunto y el otro comparativo a cualquiera de los conjuntos restantes (discriminaciones excitatorias, inhibitorias o nulas). Los resultados evidencian que en la fase de prueba, las igualaciones entre estímulos excitatorios y entre estímulos inhibitorios fueron más altas que entre estímulos nulos, conformando así, clases equivalentes de estímulos con propiedades excitatorias e inhibitorias.

En el ejemplo anterior, las relaciones de ocurrencia entre las figuras de un conjunto y el sonido mantuvieron una relación de demora corta (2 segundos), en las que la conducta de los participantes se limitó a la observación y no a su alteración o modificación.
El hecho de que el sonido fuese condicional a las ocurrencias individuales de los tres miembros de un mismo conjunto, resultó en que dichos estímulos compartieran una misma funcionalidad, de la misma manera en que la ausencia del sonido fue condicional a los miembros de otro conjunto. En otras palabras, las figuras de cada conjunto resultaron en funciones de estímulo contextualizadas como una clase equivalente, por la ocurrencia o ausencia del sonido que sirvió como evento contextualizador. Así, se puede afirmar que el comportamiento es abstracto en tanto que se estableció una propiedad funcional compartida en un conjunto de objetos, debido a una consistencia en las contingencias de ocurrencia, así como una constancia funcional de algunas de sus propiedades y una variación física de otras.

En el estudio sobre categorización de relaciones, Kotovsky y Gentner (1996) investigaron el reconocimiento de relaciones perceptuales de semejanza o de invariancia geométrica en niños, bajo un procedimiento de igualación a la muestra de dos elecciones. En la primera fase, se entrenó la relación de identidad entre imágenes de animales. En una fase de prueba, el estímulo muestra era un compuesto de tres figuras geométricas que compartían una relación de simetría (e.g., oOo) con referencia a su color o tamaño, un estímulo de comparación que compartía la misma relación (e.g., xXx) y un estímulo de comparación que no compartía tal relación (e.g., o00). Los arreglos de estímulos variaban en torno a la dimensión y cambio de polaridad, a saber: (a) misma dimensión, misma polaridad ( $\mathrm{XXx}$, oOo); (b) misma dimensión, diferente polaridad (xXx, OoO); (c) diferente dimensión, misma polaridad ( $x X x, 121) ; y$ (d) diferente dimensión, diferente polaridad ( $x X x, 212)$; las tres primeras constituían relaciones de similitud de bajo nivel y la última constituía una de alto nivel. Entre los ensayos de prueba, se presentaron ensayos de relleno con baja dificultad en los cuales se presentaban arreglos de igualación de menor complejidad (similitud por objetos compartidos), de relaciones de similitud de bajo nivel o de alto nivel, con el fin de familiarizar a los sujetos con la tarea y eliminar a aquellos que cometieran más de un error. Los resultados muestran que los niños de diferentes edades fueron capaces de reconocer las relaciones de alto nivel cuando 
se basaban en relaciones de bajo nivel, pero solo los niños de 6 y 8 años pudieron reconocer las relaciones de alto nivel sin soporte de las relaciones de bajo nivel.

En este estudio, en la fase de entrenamiento se presentaba de forma concurrente dos figuras idénticas y una diferente, con parámetros espacio-temporales constantes y teniendo en cuenta dos aspectos: (a) una variación entre ensayos en los estímulos muestra y de comparación; y (b) una constancia en la relación de igualdad en sus propiedades físicas. La constancia en la ocurrencia de dos figuras idénticas en cada ensayo, resultó en el establecimiento de una contingencia de función en la cual, la figura que se ubicaba como estímulo muestra contextualizaba a una de las figuras que servía como comparación, en términos de la igualdad en sus propiedades físicas, de tal manera, que el participante respondía diferencialmente a la similitud constante en el patrón de estímulos, ante la variación tanto de la dimensión como de la polaridad de cambio entre el estímulo estándar y los de comparación. Dado que la relación entre el estímulo estándar y el estímulo de elección idéntico o similar no dependía de ninguna respuesta operativa por parte del participante, la relación de contextualización se estableció por la constancia en los parámetros espacio-temporales de su ocurrencia y entre sus propiedades concretas. El comportamiento abstracto en este ejemplo se caracteriza por responder de forma diferencial al patrón de relación perceptual entre figuras geométricas del estímulo de comparación, en función del patrón de relación perceptual entre las figuras del estímulo muestra, ante la variación entre ensayos de las propiedades físicas y de los patrones perceptuales no contingentes.

\section{Abstracción en función suplementaria.}

El comportamiento suplementario abstracto, por su parte, se identifica con una estructura contingencial caracterizada por el establecimiento de contingencias de función derivadas del contacto con propiedades funcionales absolutas y propiedades físico-químicas variantes en los segmentos de estímulo condicionales a una respuesta operativa, entre arreglos variantes. Las contingencias de ocurrencia se caracterizan por: (a) una inconstancia entre propiedades absolutas de los estímulos contextualizadores y contextualizados que es afectada por algún segmento de respuesta; (b) una alteración en los parámetros espacio-temporales de los segmentos de estímulo contingente a una respuesta operativa; (c) un responder a la constancia de una propiedad funcional en los objetos de estímulo relativo a la variación en otras propiedades no contingentes en los mismos objetos (Ribes, 2010).

Las contingencias de función se describen por la operación de los componentes de la función de respuesta sobre los componentes de la función de los estímulos, de tal forma que la contextualización funcional de un segmento de estímulo depende de un segmento de respuesta que adicione o sustraiga el evento de estímulo contextualizador y su respuesta vinculada. El ajuste se da en la forma de una respuesta efectiva para alterar la ocurrencia del evento contextualizador, relativa a los cambios espacio-temporales de los objetos de estímulo contextualizados, los cuales tienen como característica, una constancia total respecto de sus propiedades funcionales (Ribes, 2010; Ribes \& López, 1985).

Un ejemplo de esto, se encuentra en el estudio de identificación de atributos en el logro de conceptos. Bourne y Bunderson (1963) mostraron a estudiantes de un curso de psicología elemental a una serie de figuras que variaban en dos propiedades de las modalidades de color, forma, número, tamaño, orientación, posición horizontal y posición vertical. La combinación de las dos propiedades dentro de dos modalidades (e.g., rojo-una figura, rojo-dos figuras, verde-una figura y verde-dos figuras) llevó al establecimiento de cuatro contingencias; al presentarse una figura, el sujeto recibía retroalimentación diferencial después de seleccionar uno de cuatro botones, cada uno asociado a una combinación particular. Los autores variaron: (a) la cantidad de modalidades no relevantes a la retroalimentación (2 y 5); (b) el intervalo entre estímulos (0, 4 y 8 segundos); y (c) el intervalo entre ensayos (1, 5 y 9 segundos), y tomaron como criterio del logro del concepto que se presentaran 32 ensayos correctos de manera consecutiva. En todos los casos, los conceptos fueron identificados sin aparente afecto de la demora de la retroalimentación, pero con un 
favorecimiento del incremento del intervalo entre ensayos en disminuir el número promedio de errores; este efecto fue más evidente con cinco dimensiones irrelevantes que con una.

En otro estudio, cuatro palomos fueron enfrentados con combinaciones de propiedades que definen conceptos polimorfos (Jitsumori, 1993). La combinación de propiedades implicó tres modalidades con dos propiedades cada una: triángulo y círculo para forma, negro y blanco para color de la forma, y rojo y verde para el color de fondo de la forma. En este caso, se definieron tres propiedades positivas diferentes para cada sujeto (e.g., círculo-negro-sobre rojo), así como la regla genérica dos-de-tres propiedades presentes en una imagen (e.g., triángulo-negro-sobre rojo, o círculo-blancosobre rojo) contingente a reforzamiento, y la regla uno-de-tres contingente a extinción. En la fase de entrenamiento las imágenes podían contener 3,40 5 formas iguales con disposición espacial diferente, en la primera fase de transferencia se presentaron las mismas propiedades pero se usaron diferentes disposiciones espaciales de las formas; aquí, las mayores tasas de respuesta fueron controladas por estímulos con dos o más propiedades positivas, que por estímulos con sólo una o ninguna propiedad positiva. En la segunda prueba de transferencia, se cambió una de las propiedades para cada modalidad (e.g., gris reemplazo a blanco o a negro como color de la forma); las tasas de respuesta fueron en decremento de acuerdo con la distribución del valor de la propiedad: dos positivas-una novedosa, seguidas de una-positiva-una negativa-una novedosa, y seguidas de dos negativas-una novedosa.

En los ejemplos anteriores las relaciones de ocurrencia involucraron contingencias en las que la respuesta a un conjunto de figuras y su retroalimentación o reforzamiento diferencial, fue contingente a propiedades en combinación, presentes en aquellas figuras; en cualquier caso los parámetros espacio-temporales fueron constantes (aquellas del experimento que no fueron críticas). El hecho de que la respuesta fuese retroalimentada diferencialmente ante las ocurrencias individuales de las figuras, de acuerdo a la combinación de propiedades presentes en diferentes objetos, resultó en clases diferenciadas de objetos en los que estas propieda- des establecían el momento y lugar para un tipo de responder pertinente a la situación, demarcada por consecuencias determinadas; en otras palabras, los objetos conformaron clases de estímulo dado que propiedades particulares presentes en ellos, fueron contextualizadas como una clase funcional que es relativa a la ocurrencia diferencial de la retroalimentación que fungió como evento contextualizador.

El responder abstracto implicado en esta estructura contingencial, al igual que en el responder abstracto en estructuras contextuales, se refiere a la separación de propiedades funcionales compartidas en un conjunto de objetos, en una situación que presenta una constancia tanto física como funcional de ciertas propiedades, y una variación física de las demás propiedades individuales, con la diferencia de que en el caso presente, la respuesta del organismo es operativa sobre los objetos en los que ocurre dicha constancia y variación. Es claro que no se responde a totalidades de objetos específicos, en tanto configuraciones de las mismas propiedades, si no que se extraen combinaciones críticas entre propiedades funcionales presentes en una diversidad de objetos.

\section{Abstracción en función selectora.}

En el comportamiento selector abstracto, las contingencias de función se establecen a través del contacto con propiedades relativas y variantes en los segmentos de estímulo contextualizados condicionales a una respuesta operativa, entre arreglos variantes. Las contingencias de ocurrencia se caracterizan por: (a) la presencia de un segmento de estímulo selector, adicional a la configuración de los segmentos de estímulo contextualizado y contextualizador; (b) una inconstancia entre propiedades absolutas de los estímulos contextualizadores y contextualizados que es afectada por algún segmento de respuesta operativa; (c) una alteración en los parámetros espacio-temporales de los segmentos de estímulo contextualizadores y contextualizados contingente a una respuesta operativa; (d) una permutación momento a momento en las propiedades físicas de los segmentos de estímulo contextualizados, condicional a la constancia en al menos dos valores de los segmentos de estímulo contextualizadores y selector (Ribes \& López, 1985). 
Las contingencias de función se describen por la precisión en el responder operativo, condicional tanto a las propiedades relacionales del segmento selector, como a las propiedades funcionales del segmento contextualizador. Así, la funcionalidad de los segmentos selector y contextualizado, depende de su relación contingente con las propiedades funcionales del segmento contextualizado, de tal forma que el organismo responde a propiedades relativas entre estímulos y no a propiedades absolutas. El ajuste se da en la forma de respuesta precisa ante diferentes relaciones entre propiedades de los estímulos a los que se responde, por lo que a diferencia de los casos anteriores, los objetos mantienen variabilidad relativa respecto de sus propiedades físico-químicas, y variabilidad total respecto de sus propiedades funcionales. La respuesta está desligada de las propiedades individuales y absolutas en las ocurrencias de los estímulos, por lo que las propiedades alteradas en tiempo y espacio, adicionalmente, presentan permutación en sus propiedades funcionales en relación a las ocurrencias de respuesta (Ribes, 2010).

Un ejemplar de dicha estructura contingencial se encuentra en un estudio de Robinson (1955), quien enfrentó a chimpancés a problemas de relaciones igual/diferente ejemplificados por triángulos rojos, círculos azules y cuadrados negros. En la fase de entrenamiento los sujetos debían elegir la relación igual o diferente que conformaron cuatro problemas presentados en bloques consecutivos: (a) triángulo-rojo y triángulo-rojo versus círculo-azul y cuadrado-negro; (b) círculo-azul y círculo-azul versus círculo-azul y cuadrado-negro; (c) cuadrado-negro y cuadrado-negro versus círculo-azul y triángulo-rojo; y (d) nueve combinaciones de triángulo-rojo, círculoazul, y cuadrado-negro. La elección de la relación de identidad siempre fue positiva, recompensada con comida, mientras que la elección de la relación negativa siempre fue la de diferencia, castigada con el retiro de la bandeja de respuesta. Después de alcanzar los criterios de efectividad, se realizó una prueba en la que se presentaron ensayos con los objetos de entrenamiento mezclados con objetos novedosos que correspondían a herramientas, utensilios de cocina, juguetes y artículos de baño que ejemplificaban las relaciones igual/diferente. El porcentaje promedio para los objetos de entrenamiento fue $83.3 \%$ y de $77.5 \%$ para los objetos novedosos, lo cual no implicó diferencias significativas.

En un estudio sobre la adquisición de comportamiento conceptual, Ribes, Moreno y Martínez (1998), utilizaron una tarea experimental de igualación a la muestra de segundo orden, compuesta por tres fases, cada una precedida por instrucciones específicas: (a) una fase de familiarización con la tarea; (b) una fase de entrenamiento en la que se presentaba a diferentes grupos, arreglos de estímulos con una secuencia de instancias aisladas, con segmentos de estímulo diferenciados por su orden de aparición, o con la presentación del total de la configuración del arreglo. La respuesta de igualación consistía en la selección de textos relacionados con los estímulos específicos presentes, con las modalidades pertinentes sobre las que variaban los estímulos (forma y color), o con la relación de igualación vigente (igual/diferente), con presentación de retroalimentación en cada ensayo. Para la fase de prueba se utilizaron tres pruebas de transferencia (extrainstancia, extramodal y extrarelacional) con el mismo requerimiento de respuesta pero sin retroalimentación, bajo el supuesto de que cada una de ellas evaluaba niveles de abstracción diferenciales y de complejidad progresiva. Los resultados evidencian que el comportamiento conceptual evaluado en las fases de transferencia no fue afectado por la forma de presentación de los estímulos en la fase de entrenamiento ni por las instrucciones proporcionadas, sino por la construcción de la descripción verbal en cada ensayo.

En los ejemplos anteriores, las relaciones de ocurrencia involucraron contingencias en las que la respuesta a un conjunto de figuras y su retroalimentación fue contingente a la relación entre propiedades de los diferentes objetos, ya fuera demarcada por ellos mismos, o por a la relación entre objetos que no fueron parte del conjunto; en cualquier caso los parámetros espacio-temporales fueron constantes (aquellos del experimento que no fueron críticas). El hecho de que la respuesta fuese retroalimentada diferencialmente, no ante ocurrencias individuales con propiedades singulares o en combinación, sino de acuerdo con la relación entre propiedades que sostienen los diferentes objetos, resultó en clases diferenciadas de objetos en 
relación que establecen el momento y lugar para un tipo de responder pertinente a la situación, demarcada por consecuencias determinadas; en otras palabras, las propiedades de los objetos conformaron clases de relaciones contextualizadas como correspondencias, las cuales son relativas a otras clases funcionales de propiedades de los objetos, debido a la ocurrencia diferencial de la retroalimentación que fungió como evento contextualizador.

El responder abstracto implicado en esta estructura contingencial, se refiere a la separación de propiedades funcionales en relaciones compartidas por un conjunto de objetos, cuando la situación presenta una constancia en la correspondencia entre propiedades y una variación de las propiedades individuales -tanto sobre las que se establece la correspondencia como sobre las que no- relativas a las acciones operativas del organismo. Es claro que no se responde a totalidades de objetos específicos ni a propiedades constantes, si no que se extraen correspondencias críticas entre propiedades presentes en una diversidad de objetos.

En cada uno de los niveles anteriormente descritos, ha sido posible identificar niveles diferenciales de comportamiento abstracto con base en estructuras contingenciales que se diferencian en términos de las relaciones diacrónicas y sincrónicas entre los eventos de estímulo y de respuesta implicados. Operacionalmente, la identificación del establecimiento de las contingencias de ocurrencia y principalmente de las contingencias de función, es posible a través de medidas del tipo de ajuste logrado a los criterios especificados por la circunstancia y condiciones experimentales.

La evaluación y medición del comportamiento abstracto, supone poner a prueba las nuevas funcionalidades en los segmentos de respuesta en situaciones en la cuales las contingencias de función son las mismas, pero las contingencias de ocurrencia varían. La forma usual de realizar tal evaluación se da en arreglos estimulares variables en los cuales se suprime el evento contextualizador, con el objetivo de identificar una tendencia en el responder ante objetos de estímulo que comparten propiedades funcionales con aquellos implementados en las contingencias de ocurrencia, permitiendo así, el uso del término abstracción (comportamiento abstracto) como categoría disposicional, refiriéndose a las regularidades en la reactividad del organismo ante variaciones en las propiedades de los estímulos en relación a una propiedad funcional constante sobre la cual se estableció la contingencia de función, o ante la variación en la morfología general de los eventos de estímulo, pero manteniendo constante la estructura contingencial. Así, según Ryle (1949) y Ribes (1990), los términos referidos a capacidades (disposiciones o propensiones) comprenden expresiones que identifican la inclinación o probabilidad de que ocurran ciertos actos con base en la historia de un individuo y de las circunstancias de interacción, pero que no corresponden directamente con los actos concretos que definen dichas circunstancias.

\section{Conclusión}

En el presente escrito se puso de manifiesto que el concepto de abstracción, y en sí el comportamiento abstracto, se ha usado en la tradición conductual como equivalente al concepto de conducta conceptual, referido al logro en el responder de manera semejante a una clase de objetos que comparten propiedades en común, de tal forma que el comportamiento abstracto se ha descrito como conducta bajo control del estímulo en la que la reactividad de un organismo se generaliza intra clases y se discrimina entre clases (Keller \& Schoenfeld, 1950). Esta posición puede ser caracterizada por cuatro supuestos.

Primero, una clase de estímulos está compuesta por las instancias que ejercen control discriminativo sobre una clase de respuesta con base en una propiedad funcional (Catania, 2007; Stemmer, 1980; Tonneau, 2001). Segundo, el responder discriminativo se presenta ante una propiedad constante ante la variación en otras propiedades de los estímulos que conforman la clase, como resultado de la exposición a contingencias de reforzamiento diferencial (Catania, 2007). Tercero, el responder a los estímulos de una clase puede y suele ampliarse vía generalización a nuevos estímulos de acuerdo con la propiedad discriminativa que delimita la clase (Stemmer, 1980). Finalmente, el responder puede configurarse en diferentes niveles de control de 
estímulos, teniendo como criterio de su clasificación la morfología de las propiedades discriminadas (Herrnstein, 1990; Zentall et al., 2002; Zentall, et al., 2008).

El problema con esta concepción es su carácter operacional que centra la descripción del comportamiento abstracto en los procedimientos y los resultados observados; en los primeros se resalta el aspecto de las propiedades de los objetos o de las consecuencias correlacionadas con ellas, y en los segundos se resalta el responder discriminado respecto de tales propiedades. Estos análisis dejan de lado la pregunta por los procesos de desarrollo del comportamiento abstracto, y el tipo de relación cualitativa que se establece entre las propiedades funcionales que se extraen en la circunstancia interactiva respecto de la conducta. De esta manera, los criterios de clasificación del responder abstracto se basan en la morfología de las propiedades compartidas que adquieren función discriminativa o en las consecuencias compartidas presentes en las contingencias de reforzamiento, y los procesos por los que surge se reducen a relaciones propiedadrespuesta-reforzador o propiedad-reforzador en un mismo nivel horizontal.

En el presente documento también se presentó la tesis de que el comportamiento abstracto se identifica con un responder de forma independiente respecto de la concreción o particularización de objetos o de relaciones entre sus propiedades, los cuales pueden tener funciones individuales en tanto totalidad o singularidad. El comportamiento abstracto surge cuando la situación implica la reactividad a la constancia de ciertas propiedades funcionales compartidas por un conjunto de objetos y relaciones entre objetos, ante la variación de propiedades no relevantes en las relaciones de condicionalidad prescritas.

La propuesta de Ribes y López (1985) y Ribes (2010) sobre una taxonomía que establece niveles de conducta, permite establecer diferencias cualitativas de comportamiento abstracto a partir de los cuales se pueden delimitar procesos molares en los que puede surgir la abstracción, cuando se establecen contingencias de ocurrencia y de función. Así, el responder de manera independiente a propiedades funcionales de las totalidades o singularidades de los objetos, implica el establecimiento de nuevas relaciones funcionales, o contingencias de función, que pueden organizarse de maneras cualitativamente diferentes de acuerdo a cómo un elemento es crítico para la organización de las ocurrencias de los objetos individuales. Es decir, pueden existir diferentes relaciones o estructuras contingenciales de carácter abstracto, en donde las contingencias de ocurrencia tienen la peculiaridad de que en la situación, las ocurrencias individuales presenten una constancia en ciertas propiedades físicas (individuales o en relación) de los objetos, y una variación en el remante de sus propiedades.

Con base en esto, es posible identificar niveles de interacción abstracta más o menos complejos resultantes de diferentes condicionalidades de ocurrencia y de función. En un nivel contextual de comportamiento abstracto, el responder se separa de las propiedades funcionales de totalidades o singularidades y se integra funcionalmente en una propiedad funcional compartida en un conjunto de objetos, debido a una consistencia en las contingencias de ocurrencia, así como una constancia funcional de algunas de sus propiedades y una variación física de otras. El nivel suplementario de responder abstracto, también, se refiere a la separación de propiedades funcionales compartidas en los objetos a partir de la constancia física y funcional de ciertas propiedades y la variación física de otras, cuando la respuesta del organismo es operativa sobre los objetos en los que ocurre dicha constancia y variación. Por último, en el nivel selector de conducta abstracta, la respuesta implica una separación de propiedades funcionales en relación que son compartidas por un conjunto de objetos, cuando la situación presenta una constancia en la correspondencia entre propiedades y una variación de las propiedades individuales - tanto sobre las que se establece la correspondencia, como sobre las que no - relativas a las acciones operativas del organismo. Los fenómenos conductuales descritos en la tradición de la teoría del condicionamiento, bajo el nombre de conceptualización, categorización y formación de conceptos, como equivalentes del término abstracción, pueden ser ubicados en estos tres primeros niveles interacción psicológica. 
Finalmente, la independencia del responder a totalidades o singularidades que resulta de un tipo particular de organización de las contingencias abstractas, se muestra como la tendencia o disposición a presentarse ante nuevas situaciones determinadas por variaciones en los objetos de estímulo, respecto de aquellos bajo los cuales se establecieron las contingencias prescritas. Esto ha sido recurrente en la evaluación de pruebas de generalización, trasposición o transferencia en que se examinan las nuevas funcionalidades en los segmentos de respuesta en situaciones en las cuales las contingencias de función son las mismas, pero las contingencias de ocurrencia varían.

\section{Referencias}

Bourne, L. E., \& Bunderson, C. V. (1963). Effects of delay of informative feedback and length of post-feedback interval on concept identification. Journal of Experimental Psychology, 65(1), 1-5. DOI: 10.1037/h0046994

Catania, A. C. (2007). Learning. Cornwall-onHudson, NY: Sloan.

Goldiamond, I. (1966). Perception, language, and conceptualization rules. En B. Kleinmuntz (Ed.), Problem solving: Research, method and theory (pp. 183-224). New York: Wiley.

Gutiérrez, M., \& Benjumea, S. (2003). Formación de clases funcionales utilizando un entrenamiento de condicionamiento clásico. Revista Latinoamericana de Psicología, 35(2), 165-174.

Hayes, S. C., Barnes-Holmes, D., \& Roche, B. (Eds.). (2001). Relational Frame Theory: A Post-Skinnerian account of human language and cognition. New York: Plenum Press. DOI: $10.1007 / \mathrm{b} 108413$

Herrnstein, R. J. (1990). Levels of stimulus control: A functional approach. Cognition, 37(1-2),133-166. http://dx.doi. org/10.1016/0010-0277(90)90021-B

Huber, L., \& Aust, U. (2006). A Modified Feature Theory as an account of pigeon visual categorization. En E. A. Wasserman \& T. R. Zentall
(Eds.), Comparative cognition: Experimental explorations of animal intelligence (pp. 325342). Oxford: Oxford University Press. DOI: 10.1002/9781118133880.hop203018

Hull, C. L. (1920). Quantitative aspects of the evolution of concepts. Psychological Monographs, XXVIII(1.123), 1-86.

Jitsumori, M. (1993). Category discrimination of artificial polymorphous stimuli based on feature learning. Journal of Experimental Psychology: Animal Behavior Processes, 19(3), 244-254. DOI: 10.1037/0097-7403.19.3.244

Keller, F. S., \& Schoenfeld, W. N. (1950). Principles of psychology: A systematic text in the science of behavior. New York: Appleton-Century Crofts.

Kotovsky, L., \& Gentner, D. (1996). Comparison and categorization in the development of relational similarity. Child Development, 67(6), 27972822. DOI: $10.2307 / 1131753$

Layng, J., Sota, M., \& Leon, M. (2011). Thinking through text comprehension I: Foundation and guiding relations. The Behavior Analyst Today, 12(1), 3-11. DOI: 10.1037/h0100706

Mackintosh, N. J. (2000). Abstraction and discrimination. En C. Heyes \& L. Huber (Eds.), The evolution of cognition (pp. 123-142). Cambridge, MA: MIT Press.

Patridge, E. (2006). Origins. A Short Etymological Dictionary of Modern English. London: Taylor \& Francis Group.

Ribes, E. (1990). Psicología General. Ciudad de México: Trillas

Ribes, E. (1994a). El análisis de la conducta humana. La morfología como enemigo público número uno. En E. Ribes, L. J. Hayes y F. López (Eds.), Psicología interconductual: Contribuciones en honor a J.R. Kantor (pp. 143-156). Guadalajara: Universidad de Guadalajara.

Ribes, E. (1994b). Skinner y la psicología: lo que hizo, lo que no hizo y lo que nos corresponde 
hacer. En E. Ribes (Coord.), B. F. Skinner in memoriam (pp. 139-174). Ciudad de México: Universidad de Guadalajara.

Ribes, E. (2000). Instructions, rules, and abstraction: A misconstrued relation. Behavior and Philosophy, 28(1), 41-55.

Ribes, E. (2003). What is defined in operational definitions? The case of operant psychology. Behavior and Philosophy, 31(1), 111-126.

Ribes, E. (2004). Acerca de las funciones psicológicas: un post-scriptum. Acta Comportamentalia, 12(2), 117-127.

Ribes, E. (2006). Categorías, conceptos y conducta: reflexiones teóricas. Revista Latina de Pensamiento y Lenguaje, 15(1), 5-23.

Ribes, E. (2007). Estados y límites del campo, medios de contacto y análisis molar del comportamiento: reflexiones teóricas. Acta Comportamentalia, 15(2), 229-259.

Ribes, E. (2010). Teoría de la conducta 2. Avances y extensiones. Ciudad de México: Trillas.

Ribes, E. \& López, F. (1985). Teoría de la conducta. Un análisis de campo y paramétrico. Ciudad de México: Trillas.

Ribes, E., Moreno, D., \& Martínez, C. (1998). Second order discrimination in humans: The roles of explicit instructions and constructed verbal responding. Behavioural Processes, 42(1), 1-18. DOI: 10.1016/S0376-6357(97)00056-9

Ribes, E., Torres, C., Barrera, J. A., \& Ramírez, L. (1995). Efectos de la variación modal de los estímulos en la adquisición y transferencia de una discriminación condicional en humanos adultos. Acta Comportamentalia, 3(2), 115-151.

Robinson, J. S. (1955). The sameness-difference discrimination problem in chimpanzee. Journal of Comparative and Physiological Psychology, 48(3), 195-213. DOI: 10.1037/h0042463
Robinson, F. \& de Oliveira, A. (2018). Análisis crítico de la teoría del reflejo desde la propuesta teórica de la subjetividad. Diversitas: Perspectivas en Psicología, 14(1), 97-108. DOI: 10.15332/ s1794-9998.2018.0001.07

Ryle, G. (1949). The concept of mind. Londres: Hutchinson. DOI: 10.7208/chicago/9780226922 652.001.0001

Schoenfeld, W. N. (1972). Problems of modern behavior theory. Conditional Reflex, 7(1), 33-65.

Shapere, D. (1989). El problema de los términos teóricos. En L. Olivé y A.R. Pérez (Eds.), Filosofía de la Ciencia. Teoría y Observación (pp. 47-69). Ciudad de México: Siglo XXI.

Sidman, M. (1994). Equivalence relations and behavior: A research story. Boston: Authors Cooperative.

Sidman, M. (2000). Equivalence relations and the reinforcement contingency. Journal of the Experimental Analysis of Behavior, 74(1), 127146. DOI: 10.1901/jeab.2000.74-127

Sidman, M. \& Tailby, W. (1982). Conditional discrimination vs. matching to sample: An expansion of the testing paradigm. Journal of the Experimental Analysis of Behavior, 37(1), 5-22. DOI: 10.1901/jeab.1982.37-5

Skinner, B. F. (1937). Two types of conditioned reflex: A reply to Konorski and Miller. Journal of General Psychology, 16(1), 272-279. DOI: 10.1080/00221309.1937.9917951

Skinner, B. F. (1938). The behavior of the organisms. Acton Massachusetts: Copley Publish Group.

Skinner, B. F. (1957). Verbal Behavior. New York: Appleton Century Crofts. DOI: 10.10 37/11256-000

Skinner B. F. (1966). An operant analysis of problem solving. En B. Kleinmuntz (Ed.), Problem solving: Research, method and theory (pp. 225257). New York: John Wiley \& Sons. 
Stemmer, N. (1980). Natural concepts and generalization classes. The Behavior Analyst, $3(2), 41-48$.

Tonneau, P. (2001). Equivalence relations: A critical analysis. European Journal of Behavior Analysis, 2(1), 1-33.

VandenBos, G.R. (Ed.) (2007). APA Dictionary of Psychology. Washington, DC: American Psychological Association.

Zentall, T. R., Galizio, M., \& Critchfield, T. S. (2002). Categorization, concept learning, and behavior analysis: an introduction. Journal of Experimental Analysis of Behavior, 78(3), 237248. DOI: 10.1901/jeab.2002.78-237

Zentall, T. R., \& Smeets, P. M. (1996). Stimulus class formation in humans and animals. Amsterdam: Elsevier.

Zentall, T. R., Wasserman, E. A., Lazareva, O. F., Thompson, R. K., \& Ratterman, M. J. (2008). Concept Learning in Animals. Comparative Cognition \& Behavior Reviews, 3(1), 13-45. DOI: 10.3819/ccbr.2008.30002 